\title{
Improving the accuracy of automatic tennis video annotation by high level grammar
}

\author{
I Kolonias J Kittler W J Christmas $\quad$ F Yan \\ Centre for Vision, Speech and Signal Processing \\ University of Surrey \\ Guildford GU2 7XH, United Kingdom
}

\begin{abstract}
Automatic interpretation of videos in general and sports videos in particular relies heavily on the use of contextual information. The contextual information is encapsulated in form of grammars capturing the permissible relationships between the static and dynamic objects of the video content. The grammar is invariably multilevel, characterising the spatiotemporal relationships of pixels at the lowest level of analysis, and governing the interaction of objects and the evolution of concepts at the highest level of abstraction. An automatic video analysis system has been developed that applies multilevel contextual reasoning to understand and annotate tennis video. The system that exploits grammatical rules up to the point level achieves an impressive accuracy of $87.5 \%$. We demonstrate that by adding an extra level in the contextual hierarchy which imposes constraints on the evolution of tennis video at the game level, further performance improvements can be gained.
\end{abstract}

\section{Introduction}

The rapid growth of sports video databases demands novel efficient tools for sports video analysis and annotation. Such tools are required to support access to the relevant video content in video archives, but most importantly they are needed to facilitate broadcast material production and for sports event summarisation for new services offered through Internet and mobile telephone networks.

The automatic interpretation of sports videos relies heavily on the use of contextual information. The contextual information is encapsulated in the form of grammars which capture the permissible relationships between the static and dynamic objects of the video content. The grammar is invariable multilevel, characterising the spatial and temporal relationships of pixels at the lowest level of analysis, and governing the interaction of objects and the evolution of concepts at the highest level of abstraction.

A fully automatic video analysis system that applies multilevel contextual reasoning to understand and annotate tennis video has been developed. At the lowest level we exploit spatial context to establish correspondences between neighbouring video frames and construct a global view of the tennis court in terms of a mosaic of successive frames of each shot. At the next level of analysis, straight lines detected in the mosaic image are parsed through a system of spatial relational constraints to detect the tennis court structure into which all dynamic events are mapped from the respective frames to support automatic interpretation of the tennis game evolution. A grammar in the temporal dimension is used to detect and characterise the motion of dynamic objects such as the tennis ball and the players. At the next level, the key visual events, namely the spatial position of the tennis ball at the instance it hits the ground or changes direction of motion, provide input to a reasoning engine which tracks the evolution of the tennis video and outputs a summary of play. Reasoning is based on a hidden Markov model which captures the stochastic grammar governing the possible sequences of events. An annotation system that exploits grammatical rules up to the point level was reported in [7]. It was tested on the final matches of the 2003 Australian Open Championships. This system achieves an impressive accuracy of $87.5 \%$. In this paper we demonstrate that by adding an extra level in the contextual hierarchy which imposes constraints on the evolution of tennis video at the game level, further performance improvements can be gained.

The paper is organised as follows. In the next section we review the work on sport video annotation in the literature, while in Section 3 we shortly discuss the main processes comprising our tennis video annotation system. Experimental results from the application of this system and conclusions to the paper are drawn in Section 4.

\section{Related Work}

As mentioned, analysis and annotation of video databases is a very active research topic. Especially for sports videos, additional motivation stems from the fact that the development of such tools is widely acknowledged as a prerequisite for facilitating efficient access to broadcasters' ever-expanding video archives. The use of such tools enables the efficient use of archive material for production purposes or sports event summarisation for novel services 
offered through Internet and mobile telephone networks, adding great value to the archive itself. Looking at sport video annotation from a technical perspective, system designers can benefit from the definite structure that is present in sport videos in order to generate a more concise and accurate description of a given video. The grammar that describes the evolution of a sport video at the highest level is governed by the rules of the sport; this is the basis on which work on conceptual evolution tracking is developed.

For example, Mihajlovic and Petkovic [8] have developed an annotation system for Formula 1 racing videos that isolates semantic information from both the audio and the visual content of the sequences, and then infers events of semantic importance using Dynamic Bayesian Networks.

Wang et al. [15] distinguish between play and break shots in football videos by extracting colour and motion intensity features from key frames in football video sequences and using them as input to multi-modal, multi-layer models. Xie et al. [17] used dominant colour hue, ratio and motion intensity to feed a set of HMMs and infer whether the given clip is a play or a break shot.

Assfalg et al. [1] analysed a sport-related video archive, including live video feeds from the main camera, anchor persons' studio scenes and highlight collections. Recognised sports include Water sports, Gymnastics, Other indoor sports, Track and field events and Other Outdoor sports. In [2], annotating football videos via automatically detecting match highlights is performed — and highlights such as the kick-off, shots on goal, set pieces, turnovers, counter-attacks and forward passes are detected. Tjondronegoro et al. [13] both integrate highlights in the video summarisation process and utilise cues like referee whistling, crowd excitement noise or captions (such as athletes' names, scores etc.) to both facilitate fast detection of important play events and create a general content-based retrieval framework. Finally, Kijak et al. [6] analyse the structure of a tennis video using Hidden Markov Models and fusing audio and visual cue data in their reasoning scheme, in order to segment tennis videos into a set of shots classified under one of the following categories: First missed serve, Rally, Replay and Commercial Break.

Arriving at this level of abstraction though requires the recognition of elementary actions in the field of play. A large body of work has been devoted to this area as well; for example, Petkovic et al. [10] determine how a player hits the tennis ball using HMMs; hit types include forehand and backhand strokes, volleys, serves, etc. In [4], a framework analysing sequences of various sports (including tennis) was proposed. In tennis sequences, events such as serves, returns, aces and faults are detected. In [11], the authors characterise play shots of tennis video sequences as either Ace, Fault, Double Fault, Serve and Volley or Rally.

Denman et al. [3] concentrate on snooker, developing a system that summarises a snooker match on a shot-byshot basis. That is, every shot is analysed as to whether it resulted in a pot or a near miss and the decisions will be concatenated into a single frame. Football has also featured in the work of Tovinkere and Qian [14], where a rulebased system modelling football events and rules was created. Duan et al. [4] created a framework analysing sport sequences of various sports, including football - where events such as corner kicks, free kicks, penalties and goals are detected. Wang et al. [16] presented a system capable detecting events such as goals, fouls or 'Other events' using a SVM-based classification system in football videos taken from a single camera.

\section{Evolution Tracking of Tennis Videos Using Context}

In this work, the objective is to perform semantic annotation and score-keeping of tennis match videos based only on the captured visual data (i.e. not taking text boxes superimposed by the broadcaster into account). The system is intended for use with low-quality, off-air video from a single camera (unlike for example [9]). Figure 1 illustrates its basic layout - the main tasks required for this application, and the sequence in which they are carried out.

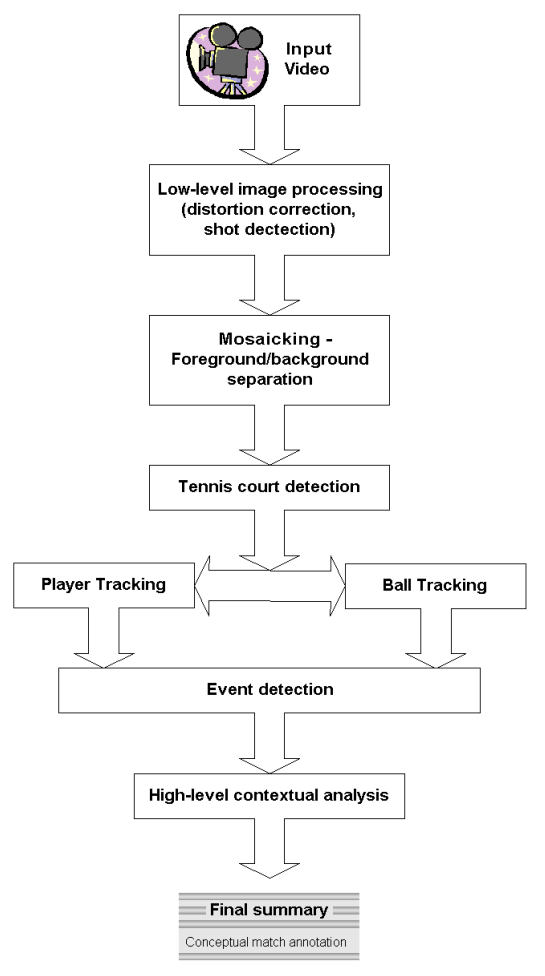

Figure 1. Sequence of detection and tracking tasks for the tennis annotation system

In this application, low-level image processing consists of reading in the video sequence, de-interlacing the frames 
and applying a preset amount of lens distortion correction. Then, by simply thresholding the difference of colour histograms between adjacent fields, shot boundaries are detected. However, more sophisticated algorithms are required to perform the remaining operations.

\section{Mosaicking and foreground separation}

Separating the foreground from the background in each frame (or field, for interlaced video) of the input sequence is crucial for the proposed tennis video annotation system. Since both foreground objects (the players and the ball) and the background (the tennis court) are required, an approach based on mosaicking [12] is the most appropriate for achieving both objectives at the same time. Each point in the observed scene is assigned a unique label, linked to its semantics (position, colour and neighbourhood relationships) on the scene; mosaicking identifies such correspondences across a shot. While the semantics of each point are not relevant in this task, correspondence labelling allows us to register consecutive frames and build a global scene view conveyed by the mosaic in the following manner:

1. Input fields are processed by a SUSAN corner detector to find a set of representative features.

2. Scene point matching based on finding the best correspondence between corner pixels detected in consecutive fields is carried out. In this case, context is conveyed in both the spatial and the temporal domain - matching features appear at the same scene positions and are located close to each other in consecutive fields of the captured video, since the camera only introduces a small amount of pan, tilt or zoom throughout the shot. This concept is illustrated in Figure 2.

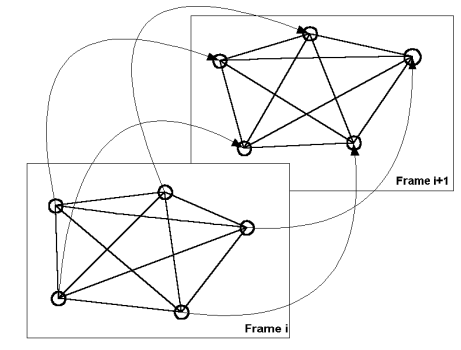

Figure 2. Spatio-temporal context in image sequences

3. After establishing inter-field homographies, shots are classified as to whether they may belong to 'play' sequences or not.

4. If the shot being processed is likely to contain a passage of play, its mosaic image is extracted by warping the fields back into the mosaic coordinate system.
Then, a simple median filtering process will provide a robust estimate of the scene background.

\section{Tennis court detection}

Once a background image has been produced, the tennis court inside the observed scene needs to be identified. For this purpose, a model graph of a tennis court is available through its description in the tennis rules [5] — therefore, the following process is followed:

1. Detect the lines in the mosaic, using a Hough Transform-based line detector.

2. Find the best match between detected mosaic lines and model tennis court ones. This is a graph matching task where only spatial context exists (the relative positions of the lines). If, at this step, the line pattern inside the mosaic image is incompatible with that of a tennis court, the shot is no longer processed.

3. Calculate a projective transformation for mapping observed events to the model court.

\section{Player tracking}

Again, the mosaic image is required; here though, individual fields are invoked as well. Player tracking consists of the following steps:

1. Candidate blobs are generated by warping each field back to the mosaic, and extracting the foreground pixels - those significantly different to the corresponding mosaic ones. These are then clustered into blobs, based on spatial connectivity, and blob statistics are extracted.

2. Player blobs should be of reasonable size, not rapidly change within the sequence, and not move very quickly around the court — while contour shapes may change quite considerably through time. They are also expected to appear at certain areas at the start of play and keep their sides during play; therefore, their movement is spatially constrained and a good initial estimate of their position is available. Thus, even a simple tracker can identify the blob corresponding to each player at any time.

\section{Tennis ball tracking}

The ball appears as an extremely small object — as little as just 5 pixels in an input field image. It is also very fastmoving, and can easily be blurred into the background, and its visibility may be further inhibited by image artefacts. Hence, ball tracking constitutes a much more challenging 
problem than player tracking - and a more applicationspecific approach is required in order to generate the appropriate features to tackle this problem. In this section, we will outline the ball tracking system presented in [18].

The ball appears as a circular (or elliptical, if it moves) blob of a bright yellow-green colour. When foreground pixels are labelled, they are clustered into blobs based on their connectedness. Then, an ellipse is fitted to each blob's boundary, and a quality measure of the fit is extracted. Finally, using shape information and blob colour, an SVMbased classifier labels blobs as either 'ball' or 'no ball'.

The blobs labelled as 'ball' by the SVM classifier satisfy the spatial context requirements for a blob to be considered as a ball; thus, false positives may still be present. These false positives may be identified based on temporal context. To this end, we exploit the dynamics of the ball to deduce its flight during play. The aim is to determine which (if any) candidate in each field is the ball.This problem is tackled in two levels - first, ball candidates are used to identify each ball flight between successive externally enforced changes in ball direction (i.e. ball hits or bounces) with a different label. These segments of ball flight (called 'trajectories' from here on) and temporal context can be exploited for tracking the ball in this formulation. As gravity is the only force exerted on the ball within a trajectory, a second-order dynamic model for predicting the ball flight is used. Secondly, candidate trajectories are linked into separate 'plays' - that is, the complete movement of the ball between breaks of play. In this case, there is both spatial and temporal context to be used - the fact that consecutive trajectories within the same play are very close in the spatio-temporal domain, whereas there is a significant gap between all other trajectories.

\section{Event detection}

The rules of tennis [5] provide a good guideline as to what events must be tracked to follow the evolution of a tennis match. Such events are the ball being hit by the players, the ball bouncing on the court, and breaks of play.

Figure 3 shows an example of the final ball tracking / event detection result, where one false positive (before the serve) and two false negatives (both far player hits) appear. Recovery from errors made in this stage is left to the highlevel reasoning engine for score keeping.

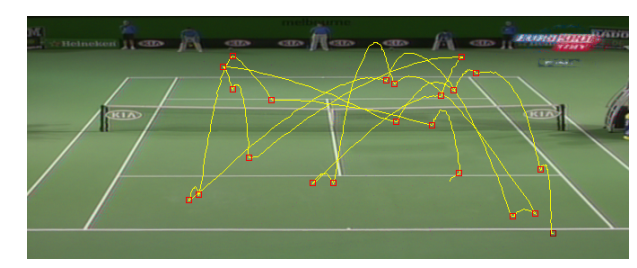

Figure 3. Tracking result with detected events superimposed on mosaic image
Having detected possible event locations in the spatiotemporal domain, we now determine the type of event that occurred. The rules of tennis stipulate that there are some events which have to be treated differently to all others - for example, the players serving. Contrary to ball event detection, there is only spatial context in this case, as both the player and the ball must be positioned within a certain range inside the court and each other. The data used to construct each observation are the blobs labelled as players in each field, the ball position and speed and the events detected in the previous step. For a serve to occur, one of the players' contours must have the position and body pose normally associated with a serve, as shown in Figure 4. Ball data is also used to generate a confidence measure of whether the ball is in a plausible serve position.
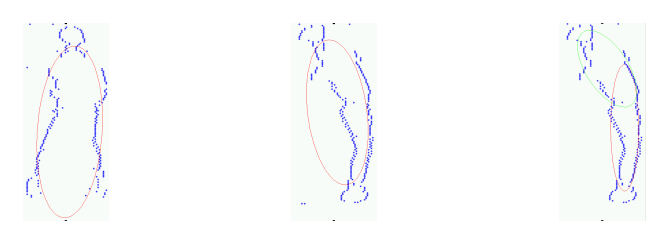

\section{Figure 4. Detecting serves via players' body poses}

Other events carrying a semantic importance in the evolution of a tennis match can now be detected and mapped onto the model court for further reasoning. The player and ball tracking data, along with the detected ball events and the mosaic image - model court mapping, are now used to compute, for all recognised ball events, a confidence measure of whether they are player hits or ball bounces. The discrimination is based on the assumption that, if a player is close to the ball and the ball is located where a hit can reasonably be made based on the player's position and contour, the event is more likely to be a hit, and therefore it is more confidently labelled as one; otherwise, it is highly unlikely to be a hit, so it is more confidently labelled as a bounce. Again, only spatial context exists.

\section{High-level contextual analysis}

Reasoning in context for sport videos exploits the fact that the rules imply a scoring scheme to determine which contestant wins - therefore, events associated with score changes are the most conceptually important within the match. In tennis, the evolution model for awarding a point given elementary events is illustrated in Figure 5, while that of awarding a game given individual points is shown in Figure 6. Since conceptually important events are already labelled, hypotheses about the match evolution can be generated - which can then be either supported or contradicted by future evidence. Thus, they will either be adopted, or abandoned in favour of more plausible interpretations.

At this level of abstraction, only temporal context exists between events and states of play. This reduces the evolu- 


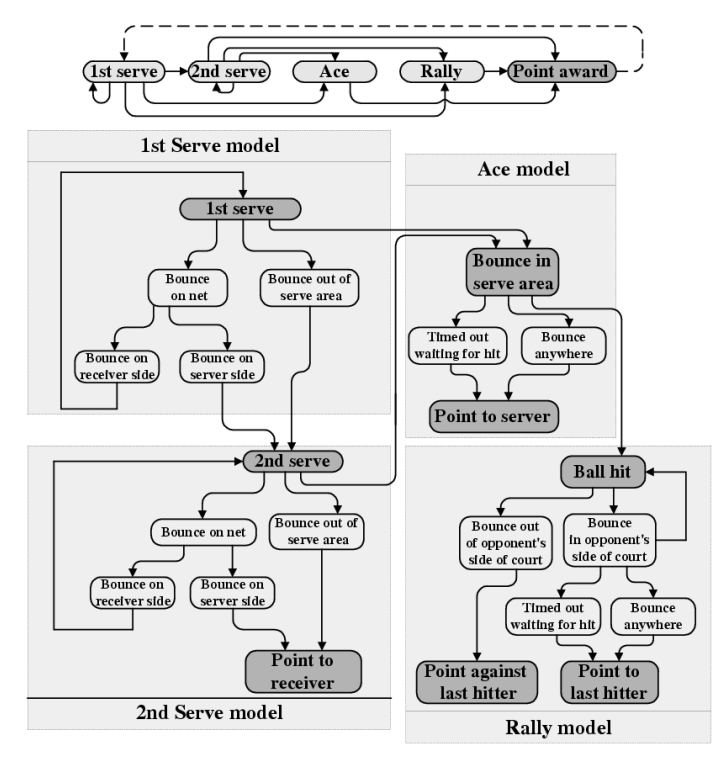

Figure 5. State models for a tennis point

tion tracking problem to associating event observations to a Markov chain containing these labels, and discovering the most likely path between them for the given observations.

Event detection errors are also bound to be encountered, and the reasoning engine should be robust to such erroneous input. To address this, HMMs with a look-ahead decision mechanism have been used, taking into account events that occur after the one currently examined to validate hypotheses generated by it. The look-ahead window length has been limited to two events, which allows reliable correction of isolated errors, and (in most cases) two consecutive errors as well - unless they occur at the end of a play. There are two reasons for this choice:

- If the look-ahead window is too long, short shots containing errors may be wrongly interpreted. Correction to such errors can only be achieved by higher levels of reasoning. For example, a wrongly awarded point will be addressed at game award level, and so on.

- Due to the difficulty in tracking the ball and the fact that it can be very hard to decide whether the ball has bounced (or where it did), ball bounces are the events most susceptible to errors. If the ball is in play, only one bounce may occur between successive hits; thus, detecting a player hit (and not serve) the ball essentially removes the chance of a point being awarded even if the bounce point is wrongly detected (or not detected at all).

\section{Annotation Results at Shot, Point and Game level - Conclusions}

The scheme described above has been tested on approximately 40 minutes of play from the Women's Final of the

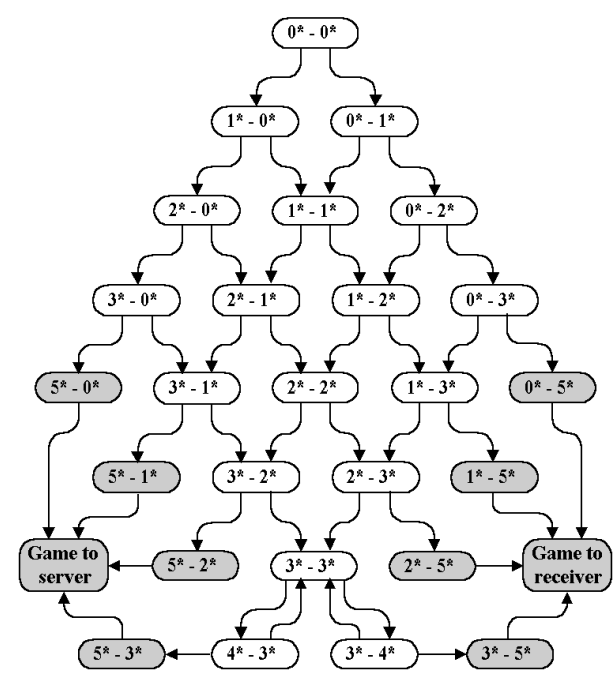

Figure 6. State model for score keeping during a game in tennis

2003 Australian Tennis Open, as well as on a little over 1 hour of the Men's Final of the same tournament. The sequences included 80 and 136 'play' shots respectively, all of which were correctly recognised in both sequences.

If a shot contains an entire play sequence, there are 5 possible outcomes: no play; bad serve by either player; point awarded to either player. Out of 80 play shots contained in the footage of the 2003 Australian Open Women's Final, 56 were correctly inferred, based on their outcome. However, when contextual information about point award was taken into account (such as the change of position for the player's serve following the award of a point, as described in [5]), this number rose quite considerably; out of the 48 points that were awarded within the 80 shots played, 42 were correctly awarded (a recognition rate of $87.5 \%$ ). For the Men's Final match of the same tournament, the footage processed contained 136 play shots, of which 93 were correctly inferred. When contextual information about point award was taken into account, an improvement in the recognition rate for points was observed again; out of the 99 points awarded in the sequence, 74 were correctly given a correct rate of $74.75 \%$. If we move on to incorporate the rules' grammar at game level, the results are mixed; while, in the Women's match, all 7 games played were correctly awarded, in the Men's match only 12 out of 19 games were correctly awarded (a rate of 63.2\%). These results are summarised in Table 1.

\begin{tabular}{l||l|l|l||l|l|l}
\multicolumn{1}{c||}{ Level of processing } & \multicolumn{3}{c||}{ Women's Final } & \multicolumn{4}{c}{ Men's Final } \\
& Correct & Total & Rate & Correct & Total & Rate \\
\hline \hline Shots & 56 & 80 & $70 \%$ & 93 & 136 & $68.38 \%$ \\
\hline Points & 42 & 48 & $87.5 \%$ & 74 & 99 & $74.75 \%$ \\
\hline Games & 7 & 7 & $100 \%$ & 12 & 19 & $63.2 \%$
\end{tabular}

Table 1. System performance at different levels 
These results reflect the current state of system development. A more detailed analysis indicates that overall improvement can be achieved by further work in these areas:

- Not enough data to train the HMM parameters in the high-level reasoning (in both point and game level) is available; the model had to be hand-crafted, based on the authors' experience as a tennis enthusiast. The model is complex, and could undoubtedly be improved. On the other hand, preliminary testing has shown that, to train the proposed model satisfactorily, one needs to present the system with an enormous volume of data, which is both difficult to handle and which the modules at the lower levels will take an extremely long time to process.

- At game level, the main reason for the mixed performance of the model is the fact that, when the system fails to award the game correctly, it does not award it at all - which clearly suggests that a bias towards undersegmenting the event chains into games is present.

- It can be difficult to identify ball bounce events sometimes the deflection of the ball in the image plane is barely perceptible. Even if the events are correctly detected, it might still be genuinely hard to determine whether a ball has bounced in or out of the court and that can make the difference in awarding points to one player or another.

- The system relies on the success of the serve detection algorithm in order to start processing a shot. If it fails, the sequence is not analysed further.

- If a shot contains more than one serve, the system currently only picks up the first one.

- Hits other than serves can be much trickier to identify - especially when the ball bounces right in front of a player ready to hit it (and thus starting to swing the racket towards the ball).

\section{References}

[1] J. Assfalg, M. Bertini, C. Colombo, and A. D. Bimbo. Semantic Annotation of Sports Videos. IEEE Multimedia, 2002.

[2] J. Assfalg, M. Bertini, C. Colombo, A. D. Bimbo, and W. Nunziati. Semantic Annotation of Sports Videos: Automatic Highlights Identification. ELSEVIER Computer Vision and Image Understanding, 2003.

[3] H. Denman, N. Rea, and A. Kokaram. Content-based Analysis for Video from Snooker Broadcasts. ELSEVIER Computer Vision and Image Understanding, 92(2-3):176-195, November-December 2003.
[4] L.-Y. Duan, M. Xu, T.-S. Chua, Q. Tian, and C.-S. Xu. A mid-level representation framework for semantic sports video analysis. In Proceedings of the eleventh ACM International Conference on Multimedia, pages 33-44, 2003.

[5] International Tennis Federation. Rules of Tennis. 2006.

[6] E. Kijak, L. Oisel, and P. Gros. Hierarchical Structure Analysis of Sport Videos Using HMMs. In Proceedings of the 2003 IEEE International Conference on Image Processing (ICIP 2003), volume 2, pages 1025-1028, September 2003.

[7] I. Kolonias, F. Yan, W. J. Christmas, A. Kostin, and J. Kittler. A contextual reasoning framework for scene interpretation and tracking in tennis video sequences. Computer Vision and Image Understanding, To appear, 2007.

[8] V. Mihajlovic and M. Petkovic. Automatic Annotation of Formula 1 Races for Content-Based Video Retrieval. Technical Report TR-CTIT-01-41, Centre for Telematics and Information Technology, Computer Science Department, University of Twente, PO BOX 217, 7500 AE Enschede, The Netherlands, 2001.

[9] C. H. N. Owens and C. Stennet. Hawk-Eye Tennis System. In Proc. VIE 2003 - Intl. Conf. on Visual Information Engineering, pages 182-185, July 2003.

[10] M. Petkovic, W. Jonker, and Z. Zivkovic. Recognizing strokes in tennis videos using Hidden Markov Models. In Proceedings of Intl. Conf. on Visualization, Imaging and Image Processing, Marbella, Spain, Sep 2001.

[11] N. Rea, R. Dahyot, and A. Kokaram. Classification and Representation of Semantic Content in Broadcast Tennis Videos. In IEEE International Conference on Image Processing (ICIP 2005), September 2005.

[12] H.-Y. Shum and R. Szeliski. Panoramic Image Mosaics. Technical Report MSR-TR-97-23, Microsoft Research, 1997.

[13] D. Tjondronegoro, Y.-P. P. Chen, and B. Pham. Integrating Highlights for More Complete Sports Video Summarization. IEEE Multimedia, 2004.

[14] V. Tovinkere and R. J. Qian. Detecting Semantic Events in Soccer Games: Towards a Complete Solution. In Proceedings of the 2001 IEEE International Conference on Multimedia and Expo (ICME'01), pages 1040-1043, 2001.

[15] F. Wang, Y.-F. Ma, H.-J. Zhang, and J.-T. Li. A Generic Framework for Semantic Sports Video Analysis Using Dynamic Bayesian Networks. In Proceedings of the 11th International Multimedia Modelling Conference, 2005 (MMM 2005), pages 115-122, January 2005.

[16] J. Wang, C. Xu, E. Chng, X. Yu, and Q. Tian. Event Detection based on non-broadcast Video. In Proceedings of the 2004 IEEE International Conference on Image Processing (ICIP 2004), volume 3, pages 1637-1640, October 2004.

[17] L. Xie, P. Xu, S.-F. Chang, A. Divakaran, and H. Sun. Structure analysis of soccer video with domain knowledge and Hidden Markov models. Pattern Recognition Letters, 25(7):767-775, 2004

[18] F. Yan, A. Kostin, W. Christmas, and J. Kittler. A novel data association algorithm for object tracking in clutter with application to tennis video analysis. In Proceedings of the IEEE Computer Society Conference on Computer Vision and Pattern Recognition, volume 1, pages 634-641. IEEE Computer Society, June 2006. 